\title{
Cognitive behavioral group therapy versus psychoeducational intervention in Parkinson's disease
}

This article was published in the following Dove Press journal:

Neuropsychiatric Disease and Treatment

\author{
Isabella Berardelli' \\ Maria Carmela Bloise ${ }^{2}$ \\ Matteo Bologna ${ }^{2,3}$ \\ Antonella Conte ${ }^{2,3}$ \\ Maurizio Pompili' \\ Dorian A Lamis ${ }^{4}$ \\ Massimo Pasquini ${ }^{2}$ \\ Giovanni Fabbrini ${ }^{2,3}$ \\ 'Department of Neurosciences, \\ Mental Health and Sensory \\ Organs, Suicide Prevention Center, \\ Sant'Andrea Hospital, Sapienza \\ University of Rome, Rome, \\ ${ }^{2}$ Department Human Neurosciences, \\ Sapienza University of Rome, Rome, \\ ${ }^{3}$ Neuromed Institute (IRCCS), Pozzilli \\ (IS), Italy; ${ }^{4}$ Department of Psychiatry \\ \& Behavioral Sciences, Emory \\ University School of Medicine, Atlanta, \\ GA, USA
}

Objective: The aim of the current study was to evaluate whether cognitive behavioral group therapy has a positive impact on psychiatric, and motor and non-motor symptoms in Parkinson's disease (PD).

Methods: We assigned 20 PD patients with a diagnosis of psychiatric disorder to either a 12-week cognitive behavioral therapy (CBT) group or a psychoeducational protocol. For the neurological examination, we administered the Unified Parkinson's Disease Rating Scale and the non-motor symptoms scale. The severity of psychiatric symptoms was assessed by means of the Hamilton Depression Rating Scale, the Hamilton Anxiety Rating Scale, the Brief Psychiatric Rating Scale, and the Clinical Global Impressions.

Results: Cognitive behavioral group therapy was effective in treating depression and anxiety symptoms as well as reducing the severity of non-motor symptoms in PD patients; whereas, no changes were observed in PD patients treated with the psychoeducational protocol.

Conclusion: $\mathrm{CBT}$ offered in a group format should be considered in addition to standard drug therapy in PD patients.

Keywords: Parkinson's disease, cognitive behavioral group therapy, psychoeducation, motor symptoms, non-motor symptoms

\section{Introduction}

Parkinson's disease (PD) is a neurological disorder mainly characterized by bradykinesia, rigidity, and tremor. The majority of PD patients also experience nonmotor and psychological symptoms, including depression, anxiety, apathy, psychosis, and impulse control disorders. ${ }^{1,2}$ Depressive and anxiety symptoms occur in up to $50 \%$ of PD patients and are also associated with poorer quality of life. ${ }^{3,4}$

Cognitive behavioral therapy (CBT) is one of the most well-established interventions in reducing both depression and anxiety. Several studies have investigated the efficacy of individual CBT on psychiatric disorders in PD., By contrast, only a handful of studies have evaluated the efficacy of CBT groups on psychiatric disorders in PD patients..$^{5-9}$ Group CBT may be useful in the adaptation process in patients affected by a chronic degenerative disease. In PD, the disease may elicit psychological reactions that are expressed in the form of phenomena of biological activation, and of subjective experiences of behavioral reactions and psychiatric symptoms (particularly anxiety and depressive symptoms). In a previous open study, in which we investigated the effect of a group CBT intervention in PD patients, we found that group CBT was useful for treating psychiatric and neurological symptoms. ${ }^{10}$ However, the efficacy of group CBT 
in PD patients needs to be confirmed by controlled studies. Currently, no study has compared the effects of group CBT with the effect produced with a psychoeducational protocol in PD patients. In view of earlier findings of the efficacy of individual and group CBT, we conducted a clinical study to investigate the effect of group CBT in PD patients and we compared the results with those obtained with PD patients treated with a psychoeducational protocol.

The primary outcome of the study was to assess the possible improvement in psychiatric symptoms as measured by several rating scales in CBT group therapy and psychoeducational intervention. The secondary outcomes were the effect of CBT group therapy and psychoeducational intervention on motor and non-motor symptoms in PD patients.

\section{Methods}

We recruited $20 \mathrm{PD}$ patients from the outpatient clinic for Movement Disorders of the Department of Neurology and Psychiatry, Sapienza University of Rome, Rome, Italy. Patients were enrolled if they had a psychiatric diagnosis based on the Structured Clinical Interview for Diagnostic and Statistical Manual of Mental Disorders (DSM)-IV Axis I Disorders (SCID-I). Exclusion criteria included comorbid psychiatric diagnoses of Axis-I psychotic disorders and bipolar disorder, as well as an education level of less than middle high school. We also excluded patients with possible substance use disorders (Figure 1). After a complete description of the study, written informed consent was obtained from all the patients. The local ethics committee approved the study (Ethical Committee Sapienza University of Rome). Participants received the study treatment at no cost. Psychiatric and neurological evaluations were performed before and after the interventions.
The diagnosis of PD was made according to the European Federation of Neurological Society and Movement Disorders Society criteria. ${ }^{11}$ The severity of PD was scored according to the Hoehn and Yahr (HY) Scale, ${ }^{12}$ the severity of motor symptoms was assessed by means of the Unified Parkinson's Disease Rating Scale, part III (UPDRS-III) ${ }^{13}$ and the quality of life was assessed using the Parkinson's Disease Quality of Life Questionnaire (PDQ8). ${ }^{14}$ Non-motor symptoms (NMS) were assessed by means of the Non-Motor Symptom Scale. ${ }^{15}$ Cognitive function was measured using the Montreal Cognitive Assessment Scale (MoCA) ${ }^{16}$ and the Frontal Assessment Battery Scale (FAB). ${ }^{17}$ To be able to conduct a reliable SCID-I, ${ }^{18}$ we only enrolled patients without evidence of cognitive impairment (MoCA score $>26$ ).

The psychiatric evaluation was performed by two trained psychiatrists and was based on the SCID-I for Axis-I disorders and the SCID-II for Axis-II disorders. The severity of psychiatric symptoms was assessed by the Hamilton Depression Rating Scale (HAM-D), ${ }^{19}$ the Hamilton Anxiety Scale (HAM-A), ${ }^{20}$ the Brief Psychiatric Rating Scale (BPRS), ${ }^{21}$ and the Clinical Global Impressions (CGI). ${ }^{22}$ To assess the severity of depressive and anxiety symptoms, we used the cut-off scores recommended for the HAM-D-7 (8-13, mild; 14-18, moderate; $\geq 19$, severe) and for the HAM-A ( $\leq 17$, mild; $18-24$, moderate; $\geq 24$, severe). All of the PD patients were diagnosed with a psychiatric disorder. Apathy was rated using the apathy evaluation scale. ${ }^{23}$

Patients were pseudorandomized to one of the two groups of treatment by matching duration and severity of Parkinson disease and homogeneity in psychiatric clinical diagnosis. In our study, 10 of the 20 PD patients were assigned to group CBT and 10 to the psychoeducational protocol

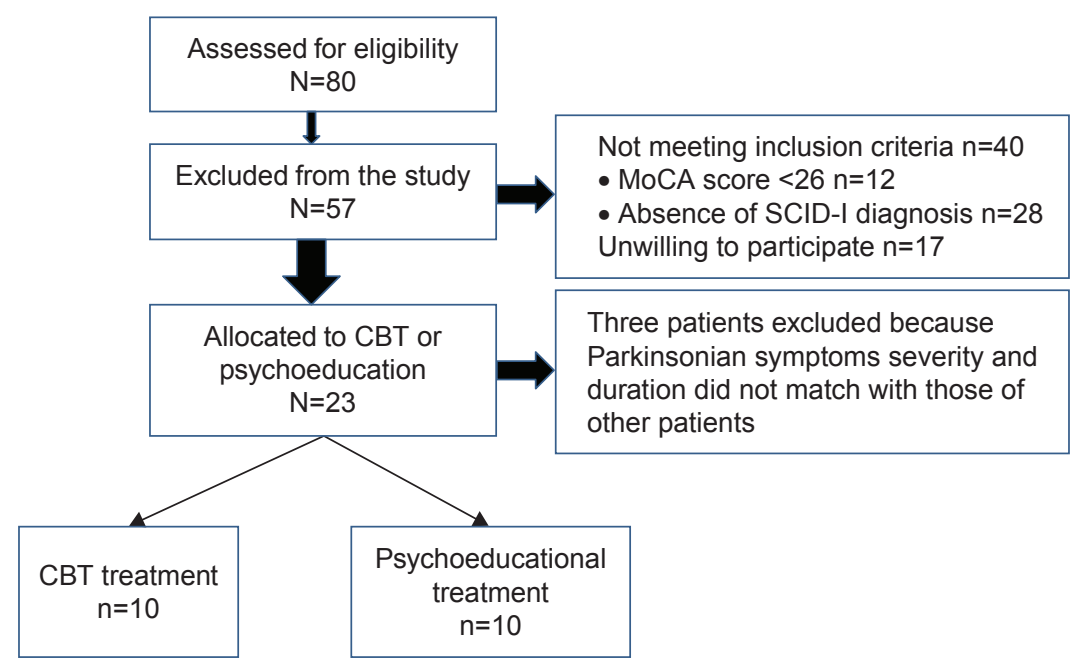

Figure I Participants' flow diagram.

Abbreviations: CBT, cognitive behavioral therapy; MoCA, Montreal Cognitive Assessment Scale; SCID, Structured Clinical Interview for DSM-IV. 
(psychoeducational group). The neurological and psychiatric evaluations were performed at baseline and at the end of the group intervention (12 weeks) in the group CBT, and at baseline and at the end of the psychoeducational intervention in the psychoeducational group (12 weeks). The baseline and final assessments were conducted by a neurologist and psychiatrist who were blind to the type of intervention.

\section{CBT group}

A CBT-trained psychiatrist and a neurologist conducted the CBT sessions. The CBT intervention consisted of 12 weekly 90-minute sessions of group therapy. ${ }^{24}$ The therapists involved in the CBT group received weekly supervision and training. The group CBT administered to the patients was standard group CBT, ${ }^{25,26}$ the only difference being that attention was paid to the link between psychological distress and physical illness. ${ }^{27,28}$ Important elements of the group CBT sessions included a convincing rationale for the intervention, training in practical skills to change mood-related thoughts, behaviors and PD motor symptoms, an encouraging practice of the skills outside the therapy sessions, and the attribution of improvement to the use of the skills. ${ }^{29} \mathrm{We}$ also evaluated specific aspects, including the tendency to avoid social situations, family conflict, and low levels of assertiveness and self-sufficiency. In addition, several sessions were devoted to problem-solving, coping strategies, and modeling. ${ }^{30}$

\section{Psychoeducational group}

The psychoeducational group met every 2 weeks. A psychiatrist and a neurologist (different from those who conducted the CBT group) conducted the psychoeducational sessions. The interventions focused on information and explanations concerning the neurological disease and the possible effects on patients' psychological world. Four main areas were targeted: illness awareness; adherence to treatment; early detection of motor and non-motor symptoms; and lifestyle regularity. Each session began with a 30-40 minute presentation on the topic of the day, followed by a related exercise and concluded with a group discussion. Although the principal goal of psychoeducation for PD was to provide accurate and reliable information regarding the disease course and therapeutic strategies, additional objectives included teaching patients' self-management skills and helping them make informed decisions about their own management within the context of a collaborative working relationship with their clinical team. Some sessions were focused on side effects of PD medication. Other sessions included an explanation of motor complications and other side effects, such as impulsive and compulsive behavior, hallucinations, and delusions. When possible, psychoeducational interventions were also personalized, for example, by taking into account the individual's unique pattern of illness, their risk factors for relapse, and their current social circumstances.

All variables are expressed as mean \pm SD. Differences in age, years of education and gender between PD patients allocated to the CBT group therapy and PD patients allocated to the psychoeducational group were evaluated by means of the Mann-Whitney U test. Non-parametric tests (ie, MannWhitney $\mathrm{U}$ test and Wilcoxon sum square test) were used to identify potential differences between the two groups in the mean baseline values of the psychiatric and neurological rating scales, and to identify any differences within each group in the psychiatric and neurological rating scale scores before and after the interventions. Bonferroni's correction was applied to multiple comparisons. All $p$-values $\leq 0.05$ were considered statistically significant. Data were analyzed using SPSS version 13.0.

\section{Results}

One of the 10 patients assigned to the group CBT, and one of the 10 patients assigned to the psychoeducational group did not complete the protocol. Therefore, we analyzed data for nine patients in the CBT group and nine patients in the psychoeducational group who participated in all the sessions of the study (Table 1).

Table I Demographic and clinical data of patients with Parkinson's disease who completed the interventions

\begin{tabular}{|c|c|c|}
\hline & $\begin{array}{l}\text { CBT group } \\
\mathbf{N}=9\end{array}$ & $\begin{array}{l}\text { Psychoeducational } \\
\text { group } \\
\mathbf{N}=9\end{array}$ \\
\hline Age (years) & $60.5 \pm 5.6$ & $57.1 \pm 5.3$ \\
\hline Gender & $6 \mathrm{M} / 3 \mathrm{~F}$ & $5 \mathrm{M} / 4 \mathrm{~F}$ \\
\hline Education (years) & $13.7 \pm 7.4$ & $12.4 \pm 7.7$ \\
\hline $\begin{array}{l}\text { Disease duration } \\
\text { (years) }\end{array}$ & $7.1 \pm 32.4$ & $5.2 \pm 2.0$ \\
\hline $\begin{array}{l}\text { Hoehn and Yahr } \\
\text { scale }\end{array}$ & All patients stage II & All patients stage II \\
\hline MoCA & $29.2 \pm 0.9$ & $28.8 \pm 1.6$ \\
\hline FAB & $17.7 \pm 0.4$ & $17.2 \pm 1.3$ \\
\hline \multirow[t]{3}{*}{ SCID-I } & $\begin{array}{l}\text { Depressive disorders: } \\
2 \text { pts }\end{array}$ & Depressive disorders: 3 pts \\
\hline & $\begin{array}{l}\text { Adjustment disorder: } \\
3 \text { pts }\end{array}$ & Adjustment disorder: 3 pts \\
\hline & Anxiety disorders: 4 pts & $\begin{array}{l}\text { Anxiety disorders: } 2 \text { pts } \\
\text { Pathological Gambling: I pt }\end{array}$ \\
\hline SCID-II & No diagnosis & No diagnosis \\
\hline
\end{tabular}

Note: Data presented as mean \pm SD.

Abbreviations: CBT, cognitive behavioral therapy; MoCA, Montreal Cognitive Assessment Scale; FAB, Frontal Assessment Battery Scale; SCID-I, Structured Clinical Interview for DSM-IV Axis I Disorders; SCID-II, Structured Clinical Interview for DSM-IV Axis II Disorders; DSM-IV, Diagnostic and Statistical Manual of Mental Disorders, version IV; F, female; M, male; pts, patients. 
Table 2 Effect of CBT and psychoeducational interventions on neurological symptoms and quality of life

\begin{tabular}{|c|c|c|c|c|c|c|}
\hline & \multicolumn{2}{|c|}{ UPDRS-III } & \multicolumn{2}{|c|}{ Non-motor symptom scale } & \multicolumn{2}{|l|}{ PDQ8 } \\
\hline & Baseline & Final & Baseline & Final & Baseline & Final \\
\hline CBT group $(\mathrm{N}=9)$ & $18.1 \pm 7.9$ & $|7| \pm 7.4$. & $21.6 \pm 15.5$ & $19.1 \pm 14.3 *$ & $7.4 \pm 4.5$ & $6.3 \pm 2.8$ \\
\hline Psychoeducational group ( $\mathrm{N}=9$ ) & $18.4 \pm 3.6$ & $19.5 \pm 5.9$ & $18.6 \pm 20.2$ & $21.1 \pm 20.2$ & $9.1 \pm 3.0$ & $10.3 \pm 3.4$ \\
\hline
\end{tabular}

Notes: No significant differences at baseline between the two groups in any of the scales used. No significant differences in scores between baseline evaluation and final evaluation in the UPDRS-III and PDQ8 scales. ${ }^{*} p=0.04 \mathrm{I}, \mathrm{z}=-2.04 \mathrm{I}$ (Wilcoxon signed rank tests). Data presented as mean \pm SD.

Abbreviations: CBT, cognitive behavioral therapy; UPDRS, Unified Parkinson Disease Rating Scale; PDQ8, Parkinson's Disease Quality of Life Questionnaire.

Patients being treated with antiparkinsonian drugs continued their treatment throughout the study, and all of the patients were examined while they were on their usual drug treatment regimen. One patient in the group CBT and one in the psychoeducational group had been on antidepressant therapy for approximately a year prior to starting the study; this medication treatment continued unchanged throughout the study.

\section{Neurological features before and after interventions}

There were no differences in UPDRS-III and NMS scores at baseline among patients who underwent the CBT treatment and those who were randomized to the psychoeducational protocol (Tables 1, $\underline{\mathrm{S} 1}$, and $\underline{\mathrm{S} 2}$ ). There were also no differences found in UPDRS scores between the two groups at the end of the two treatments (Table 2). The NMS total score improved significantly in the CBT group, but not in the psychoeducational group (Table 2). There were no significant differences before and after CBT therapy in any of the variables of the NMS scale, and only differences in the total score were significant.

\section{Psychiatric features before and after interventions}

At baseline evaluation, psychiatric diagnoses based on the SCID-I interview revealed that patients randomized to the CBT treatment and patients randomized to the psychoeducational protocol had similar psychiatric burden (Table 1). Similarly, the severity of psychiatric symptoms was also comparable between patients who participated in the CBT treatment and those who participated in the psychoeducational treatment (Table 3). The SCID-II interview did not reveal any diagnoses in either group.

The HAM-A scores, the HAM-D scores, and the BPRS scores improved significantly in the CBT group from baseline to the end of the treatment; whereas, these symptoms did not decrease among PD patients in the psychoeducational group (Table 3). The apathy score also improved significantly in the CBT group (Table 3). Regarding the CGI scores, in the CBT group at baseline, three patients had a score of 4 , and six patients had a score of 6; whereas at the end of treatment, five patients had a score of 3 , three patients a score of 2 , and one patient had a score of 0 . In the psychoeducational group, at baseline four patients had a score of 4 and five patients had a score of 3, and the scores remained unchanged at the end of the treatment. When assessing individual scores on the psychiatric rating scales and the CGI scores, we found that all participants in the CBT group improved; whereas, scores in these domains for participants in the psychoeducational group remained stable.

\section{Discussion}

The results of our study demonstrated that among PD patients in the CBT group, scores on the HAM-A decreased by $30 \%$, HAM-D scores decreased by $22 \%$, BPRS scores were reduced by $18.0 \%$, and apathy score decreased by $15 \%$, revealing that all patients treated with group CBT improved with regard to their psychiatric functioning. NMS scores were also significantly improved in the CBT group. Conversely, no changes in these measures were observed in the psychoeducational group. We also found that the

Table 3 Effect of CBT and psychoeducational interventions on psychiatric rating scales

\begin{tabular}{|c|c|c|c|c|c|c|c|c|}
\hline & \multicolumn{2}{|l|}{ HAM-A } & \multicolumn{2}{|l|}{ HAM-D } & \multicolumn{2}{|l|}{ BPRS } & \multicolumn{2}{|l|}{ Apathy } \\
\hline & Baseline & Final & Baseline & Final & Baseline & Final & Baseline & Final \\
\hline CBT group & $25.6 \pm 7.5$ & $17.9 \pm 6.0^{*}$ & $21.7 \pm 8.2$ & $17.0 \pm 6.8^{* *}$ & $34.3 \pm 3.7$ & $28.1 \pm 4.2^{*}$ & $16.4 \pm 7.6$ & $13.9 \pm 6.9 * *$ \\
\hline Psychoeducational group & $22.2 \pm 8.6$ & $22.0 \pm 8.5$ & $20.9 \pm 7.4$ & $20.7 \pm 7.2$ & $32.4 \pm 4.5$ & $32.7 \pm 4.5$ & $13.1 \pm 4.7$ & $14.7 \pm 5.3$ \\
\hline
\end{tabular}

Notes: Comparing scores obtained at the end of treatment versus scores obtained at baseline. $* p<0.01, z=-2.677 ; * * p<0.05, z=-2.546$. Data presented as mean \pm SD. Abbreviations: CBT, cognitive behavioral therapy; HAM-A, Hamilton Rating Scale for anxiety; HAM-D, Hamilton Rating Scale for Depression; BPRS, Brief Psychiatric Rating scale. 
UPDRS-III did not change significantly following the CBT group intervention or the psychoeducational group.

All the patients were diagnosed with a psychiatric disorder according to the SCID-I, and the patients' HAM-A, HAM-D, and BPRS scores were all above the cut-off and considered pathological. Therefore, it is unlikely that the differences between the CBT and the psychoeducational group were due to the variation in clinical severity of psychiatric disturbances.

The results of the present study are in keeping with those of previous studies investigating individual CBT for depression in PD patients. ${ }^{5}$ Few studies have evaluated the efficacy of group CBT for psychiatric symptoms among individuals with PD. Two such studies without a control group showed an improvement in anxiety and depression symptoms following group CBT; ${ }^{10,31}$ whereas other studies used a waitlist control group. ${ }^{32,33}$ Our study demonstrated that group CBT improves psychiatric symptoms in PD patients when compared to those receiving a psychoeducational intervention. Our study therefore confirms that group CBT improves psychiatric symptoms in PD patients also when compared with a psychoeducational intervention. The CBT group therapy began with a brief summary of the aims of the group and the rationale for learning what would be taught during the sessions. Each session focused on a specific set of skills. The first level of CBT group therapy consisted of modifying specific thoughts and behaviors. ${ }^{34}$ This level included identification of thoughts and behaviors with potentially positive or negative effects on mood, and the identification of PD motor symptoms associated with mood and anxiety changes. The second level included learning self-instructional methods, which allowed the patients to start taking over the function previously played by the therapist. ${ }^{35}$ The third level focused on logical analysis and was based on methods similar to those developed by Ellis, ${ }^{36}$ and, later, by Beck et al. ${ }^{37}$ At this level, the patients question the logic of taking certain facts, values or perspectives for granted, and consider alternative ways of interpreting their own experiences. ${ }^{38}$ Some sessions were aimed at understanding the patients' vulnerability and their fear of the future.

A further novel finding of our study is the improvement in NMS in patients treated with group CBT. NMS in PD include neuropsychiatric symptoms, autonomic dysfunction, sleep disorders, fatigue, and pain. Among patients with PD, the presence and severity of NMS is associated with poorer quality of life, nevertheless few studies have investigated the effect of specific treatments addressing these disabling symptoms. ${ }^{39}$ The finding that group CBT improved NMS scores while psychoeducational therapy did not, suggests that group CBT is a useful therapeutic approach which should be considered in the treatment of PD patients. It is important to note that NMS improved in a general way, and improvement was not due only to changes in the scores of neuropsychiatric symptoms.

In this study, we used a group therapy approach to allow patients suffering from the same neurological disorder (ie, PD) to interact with each other in an organized manner on the basis of shared experiences, fears, and expectations. In this framework, the group has a personal experience and evolution, a social space that promotes the development of relations between individuals that belong to the group. ${ }^{40}$

The therapeutic factors of group therapy are the universality, the acquisition of new information, the infusion of hope and altruism, the enhancement of socialization techniques, imitative behavior, interpersonal learning, group cohesion and the psychological adaptation to the presence of an organic neurological pathology. ${ }^{41}$ Patients with chronic neurological diseases tend to experience an increased degree of stigma, withdrawal, and social isolation, which play a significant role in both the development and persistence of psychiatric comorbidity. ${ }^{42}$ The exchange of information, the development of socializing techniques, self-disclosure, shared cognitive analysis of emotions and restructuring techniques create a sense of cohesion and belonging. ${ }^{43-47}$ The recognition by individuals within the same group of both similarities and differences enhances the development of functions as well as of metacognitive skills. In PD patients, group CBT can provide a particularly powerful sense of universality and cohesion that reduces the sense of stigma and isolation through direct social support, ${ }^{48}$ whereas, the psychoeducational program in a group setting allows patients to obtain information and share their concerns, with the attention dedicated to other patients creating a climate of shared hope.

In recent years, many studies have shown the effectiveness of psychoeducational programs for treating psychiatric disorders and have demonstrated the superiority of psychoeducational family interventions as compared to standard treatments. ${ }^{49}$ Psychoeducation is a specific type of therapy that focuses on educating patients about their disorders and ways of coping. This approach has been shown to be effective in the prevention and management of many psychiatric disorders, psychiatric symptoms in medical conditions, and neuropsychiatric disorders. ${ }^{50-52}$ The psychoeducation model used in our study was based on the psychoeducational model developed by Carkhuff. ${ }^{53}$ This model has been adapted in order to teach the interpersonal 
skills of empathy, respect, concreteness, genuineness, selfdisclosure, confrontation, for PD patients with psychiatric symptoms. Additionally, structured psychological therapies that combine psychoeducation and CBT are being increasingly used in patients diagnosed with a variety of psychiatric disorders. ${ }^{54}$ Psychoeducation is a behavioral therapeutic approach which mainly focusses on briefing the patients about their illness, problem-solving training, communication training, and self-assertiveness training. The lack of clinical improvement in our PD patients treated with the psychoeducational intervention suggests that strategies which include cognitive, affective and psychomotor components such as the group CBT intervention are more likely to change complex behavior patterns influencing mood and anxiety symptoms in PD patients than didactic interventions which focus on knowledge and concepts of illness, such as the psychoeducational program. These changes of complex behavior obtained after CBT were indeed associated with an improvement in psychiatric symptoms when compared with psychoeducation.

\section{Limitations}

One limitation of the study is that the psychoeducational treatment was not equivalent to the CBT treatment in terms of number and duration of sessions. The self-report nature of the measures used in the psychiatric evaluation is also a limitation together with the relatively small number of patients studied. This report suggests that studies on a larger population with PD are therefore warranted.

\section{Conclusion}

Our findings highlight the potential relevance of a CBT group approach for chronic and disabling illnesses such as PD. The fact that group CBT was effective in PD whereas educational intervention was not, suggests that factors intrinsic to CBT therapy, beyond "social support" are at play in improving symptoms in PD. Group CBT should be considered in addition to standard drug therapy for the treatment of mental health symptoms, particularly depression and anxiety.

\section{Author contributions}

All authors contributed toward data analysis, drafting, and critically revising the paper and agree to be accountable for all aspects of the work.

\section{Disclosure}

The authors report no conflicts of interest in this work.

\section{References}

1. Aarsland D, Larsen JP, Cummins JL, Laake K. Prevalence and clinical correlates of psychotic symptoms in Parkinson disease: a communitybased study. Arch Neurol. 1999;56(5):595-601.

2. Veazey C, Aki SO, Cook KF, Lai EC, Kunik ME. Prevalence and treatment of depression in Parkinson's disease. J Neuropsychiatry Clin Neurosci. 2005;17(3):310-323.

3. Reijnders JS, Ehrt U, Weber WE, Aarsland D, Leentjens JF. A systematic review of prevalence studies of depression in Parkinson's disease. Mov Disord. 2008;23:183-189.

4. Jones JD, Marsiske M, Okun MS, Bowers D. Latent growth-curve analysis reveals that worsening Parkinson's disease quality of life is driven by depression. Neuropsychology. 2015;29(4):603-609.

5. Berardelli I, Pasquini M, Roselli V, Biondi M, Berardelli A, Fabbrini G. Cognitive behavioral therapy in movement disorders. A review. Mov Disord Clin Pract. 2015;2(2):107-115.

6. Yang S, Sajatovic M, Walter B. Psychosocial interventions for depression and anxiety in Parkinson's disease. J Geriatr Psychiatry Neurol. 2012;25:113-121.

7. Cole K, Vaughan F. Brief cognitive behavioural therapy for depression associated with Parkinson's disease: a single case series. Behav Cogn Psychother. 2005;33:89-102.

8. Dobkin RD, Allen LA, Menza M. A cognitive-behavioral treatment package for depression in Parkinson's disease. Psychosomatics. 2006; 47(3):259-263.

9. Farabaugh A, Locascio JJ, Yap L, et al. Cognitive-behavioral therapy for patients with Parkinson's disease and comorbid major depressive disorder. Psychosomatics. 2010;51(2):124-129.

10. Berardelli I, Pasquini M, Bloise M, et al. CBT group intervention for depression, anxiety and motor symptoms in Parkinson's disease: preliminary findings. Int $J$ Cogn Ther. 2015;8(1):11-20.

11. Berardelli A, Wenning GK, Antonini A, et al. EFNS/MDS-ES/ENS [corrected] recommendations for the diagnosis of Parkinson's disease. Eur J Neurology. 2013;20(1):16-34.

12. Hoehn MM, Yahr MD. Parkinsonism: onset, progression, and mortality. Neurology. 1967;17:427-442.

13. Fahn S, Elton R. Unified Parkinson's disease rating scale. In: Fahn S, Marsden CD, Calne D, Goldstein M, editors. Recent Developments in Parkinson's Disease. Folorham Park, NJ: Macmillan Health Care Information. 1987.

14. De Boer AG, Wijker W, Speelman JD, de Haes JC. Quality of life in patients with Parkinson's disease: development of a questionnaire. J Neurol Neurosurg Psychiatry. 1996;61(1):70-74.

15. Chaudhuri KR, Prieto-Jurcynska C, Naidu Y, et al. The nondeclaration of nonmotor symptoms of Parkinson's disease to health care professionals: an international study using the nonmotor symptoms questionnaire. Mov Disord. 2010;25:704-709.

16. Nasreddine ZS, Phillips NA, Bédirian V, et al. The Montreal Cognitive Assessment, MoCA: a brief screening tool for mild cognitive impairment. J Am Geriatr Society. 2005;53(4):695-699.

17. Dubois B, Slachevsky A, Litvan I, Pillon B. The FAB: a Frontal Assessment Battery at bedside. Neurology. 2000;55(11):1621-1626.

18. American Psychiatric Association. Diagnostic and Statistical Manual of Mental Disorders (4th ed., text rev.) Washington, DC: APA; 2000.

19. Hamilton M. A rating scale for depression. J Neurol Neurosurg Psychiatry. 1960;23:56-62.

20. Hamilton M. The assessment of anxiety states by rating. Br J Med Psychol. 1959;32:50-55.

21. Overall JE, Gorham DR. The brief psychiatric rating scale. Psychol Rep. 1962;10:799-812.

22. Guy W. ECDEU Assessment Manual for Psychopharmacology, revised. Rockville, MD: National Institute of Mental Health; 1976.

23. Santangelo G, Barone $\mathrm{P}$, Cuoco $\mathrm{S}$, et al. Apathy in untreated, de novo patients with Parkinson's disease: validation study of Apathy Evaluation Scale. J Neurol. 2014;261(12):2319-2328.

24. Bieling PJ, McCabe RE, Antony MM. Cognitive Behavioural Therapy in Groups. New York: Guilford Press; 2006. 
25. Bernard H, Burlingame G, Flores P, et al. Science to Service Task Force, American Group Psychotherapy Association. Clinical practice guidelines for group psychotherapy. Int J Group Psychother. 2008;58(4): 455-542.

26. Beck AT. Cognitive Therapy and the Emotional Disorders. New York: International Universities Press; 1976.

27. Leszcz M. Introduction to special issue on group psychotherapy for the medically ill. Int J Group Psychother. 1998;48(2):137-141.

28. Leszcz M, Goodwin PJ. The rationale and foundations of group psychotherapy for women with metastatic breast cancer. Int J Group Psychother. 1998;48(2):245-273.

29. Hofmann SG. An Introduction to Modern CBT: Psychological Solutions to Mental Health Problems. Oxford, UK: Wiley-Blackwell; 2011.

30. Butler AC, Chapman JE, Forman EM, Beck AT. The empirical status of cognitive-behavioral therapy: a review of meta-analyses. Clin Psychol Rev. 2006;26(1):17-31.

31. Feeney F, Egan S, Gasson N. Treatment of depression and anxiety in Parkinson's disease: a pilot study using group cognitive behavioural therapy. Clin Psychol. 2005;9(1):31-38.

32. Okai D, Askey-Jones S, Samuel M. Trial of CBT for impulse control behaviors affecting Parkinson patients and their caregivers. Neurology. 2013;80(9):792-799.

33. Troeung L, Egan SJ, Gasson N. A waitlist-controlled trial of group cognitive behavioural therapy for depression and anxiety in Parkinson's disease. BMC Psychiatry. 2014;14:19.

34. Gabbard GO, Beck JS, Holmes J. Oxford Textbook of Psychotherapy. Oxford: Oxford University Press; 2007.

35. Meichenbaum D. Self-instructional strategy training: a cognitive prothesis for the aged. Human Dev. 1974;17:273-280.

36. Ellis A. Reason and Emotion in Psychotherapy. Oxford, UK: Lyle Stuart; 1962.

37. Beck AT, Rush AJ, Shaw BF, Emert G. Cognitive Therapy of Depression. New York: Guilford; 1979.

38. Burns DD. Feeling Good: The New Mood Therapy. Revised and updated. New York: Penguin; 1999.

39. Schrag A, Sauerbier A, Chaudhuri KR. New clinical trial for non motor manifestations of Parkinson disease. Mov Disord. 2015;30(11): 1490-1504.

40. Young-Hee Choi, Kee-Hwan Park. Therapeutic factors of cognitive behavioral group treatment for social phobia. J Korean Med Sci. 2006; 21(2):333-336.
41. Thorn BE, Kuhajda MC. Group cognitive therapy for chronic pain. J Clin Psychol. 2006;62(11):1355-1366.

42. Liotti G. Disorganized/disoriented attachment in the psychotherapy of the dissociative disorders. In: Goldberg S, Muir R, Kerr J, editors. Attachment Theory: Social, Developmental and Clinical Perspectives. Hillsdale, NJ: Analytic Press, 1995:343-363.

43. Safran JD, Segal ZV. Interpersonal Process in Cognitive Therapy. New York: Basic Books. Softcover edition, 1996, Jason Aronson, Inc.

44. Husaini BA, Cummings S, Kilbourne B, et al. Group therapy for depressed elderly woman. Int J Group Psychother. 2004;54(3):295-319.

45. Alonso A, Swiller HI. Group Therapy in Clinical Practice. Washington, DC: American Psychiatric Press, Inc.; 1992.

46. Ormont LR. The Group Therapy Experience. New York: St. Martin's Press; 1992.

47. Beck JS. Coping with Depression when you have Parkinson's Disease. Bala Cynwyd, PA: The Beck Institute for Cognitive Therapy and Research; 2000.

48. Lorentzen S, Ruud T. Group therapy in public mental health services: approaches, patients and group therapists. J Psychiatr Ment Health Nurs. 2014;21(3):219-225.

49. Conradi HJ, Bos EH, Kamphuis JH, de Jonge P. The ten-year course of depression in primary care and long-term effects of psychoeducation, psychiatric consultation and cognitive behavioral therapy. $J$ Affect Disord. 2017;217:174-182.

50. Donker T, Griffiths KM, Cuijpers P, Christensen H. Psychoeducation for depression, anxiety and psychological distress: a meta-analysis. BMC Med. 2009;7:79.

51. Nussey C, Pistrang N, Murphy T. How does psychoeducation help? A review of the effects of providing information about Tourette syndrome and attention-deficit/hyperactivity disorder. Child Care Health Dev. 2013;39(5):617-627.

52. Wiseman H, Mousa S, Howlett S, Reuber M. A multicenter evaluation of a brief manualized psychoeducation intervention for psychogenic nonepileptic seizures delivered by health professionals with limited experience in psychological treatment. Epilepsy Behav. 2016;63:50-56.

53. Colijn S, Hoencamp E, Snijders HJA, van der Spek MWA, Duivennoorden HJ. A comparison of curative factors in different types of group psychotherapy. Int J Group Psychother. 1991;41:365-378.

54. Swartz HA, Swanson J. Psychotherapy for bipolar disorder in adults: a review of the evidence. Focus. 2014;12(3):251-266.
Neuropsychiatric Disease and Treatment

\section{Publish your work in this journal}

Neuropsychiatric Disease and Treatment is an international, peerreviewed journal of clinical therapeutics and pharmacology focusing on concise rapid reporting of clinical or pre-clinical studies on a range of neuropsychiatric and neurological disorders. This journal is indexed on PubMed Central, the 'PsycINFO' database and CAS,

\section{Dovepress}

and is the official journal of The International Neuropsychiatric Association (INA). The manuscript management system is completely online and includes a very quick and fair peer-review system, which is all easy to use. Visit http://www.dovepress.com/testimonials.php to read real quotes from published authors. 